\title{
Ethnologies
}

On Historicizing Epistemology. An Essay. Par Hans-Jörg

Rheinberger (Stanford, Stanford University Press, 2010 [2007].

Pp. 128. Coll. "Cultural Memory in the Present ". Traduit de

l'allemand par David Fernbach. ISBN 0-8047-6289-9)

\section{Patrick-Michel Noël}

Volume 32, numéro 1, 2010

Jouer

Play

URI : https://id.erudit.org/iderudit/045225ar

DOI : https://doi.org/10.7202/045225ar

Aller au sommaire du numéro

Éditeur(s)

Association Canadienne d'Ethnologie et de Folklore

ISSN

1481-5974 (imprimé)

1708-0401 (numérique)

Découvrir la revue

Citer ce compte rendu

Noël, P.-M. (2010). Compte rendu de [On Historicizing Epistemology. An Essay.

Par Hans-Jörg Rheinberger (Stanford, Stanford University Press, 2010 [2007].

Pp. 128. Coll. "Cultural Memory in the Present ». Traduit de l'allemand par

David Fernbach. ISBN 0-8047-6289-9)]. Ethnologies, 32(1), 232-238.

https://doi.org/10.7202/045225ar d'utilisation que vous pouvez consulter en ligne. 
discussion is exactly that, creating a conclusion that is relevant to scholars while keeping the people of the islands themselves as the ones to receive focus.

Nicholas Hartmann

Memorial University of Newfoundland

On Historicizing Epistemology. An Essay. Par Hans-Jörg Rheinberger (Stanford, Stanford University Press, 2010 [2007]. Pp. 128. Coll. "Cultural Memory in the Present ». Traduit de I'allemand par David Fernbach. ISBN 08047-6289-9)

L'épistémologie et l'histoire entretiennent une "réciprocité engageante », pour reprendre l'expression employée par Dominique Lecourt dans L'Épistémologie historique de Gaston Bachelard. L'histoire, comme discipline, peut être l'objet d'une analyse épistémologique. L'épistémologie, comme philosophie des sciences ou théorie de la connaissance (gnoséologie), peut être l'objet d'une analyse historique plus souvent qu'autrement effectuée par des philosophes. On Historicizing Epistemology s'inscrit dans la seconde déclinaison en s'interrogeant sur un « decisive moment » (1) de la philosophie des sciences au XX $\mathrm{X}^{\mathrm{e}}$ siècle, l'historicisation de l'épistémologie entendue comme réflexion sur les conditions de la connaissance scientifique. L'historicisation de l'épistémologie vient complexifier la relation histoire-épistémologie. L'histoire est non seulement objet de l'épistémologie, elle est aussi une de ses approches pour aborder l'ensemble des sciences. Une approche importante selon Anastasios Brenner qui, dans un article la Revue de métaphysique et de morale auquel Rheinberger ne se réfère pas, soutient que « l'évolution de l'épistémologie a conduit à donner à l'histoire plus de substance » (2006: 11).

L'ouvrage se présente comme un parcours chronologique retraçant le développement de l'historicisation de l'épistémologie. L'auteur soutient que cette historicisation a pour source principale la révolution scientifique par laquelle la physique classique se transforma en physique relativiste au tournant du XXe siècle. C'est de l'activité scientifique elle-même, ou plutôt de ses praticiens, qu'est venue l'impulsion de l'épistémologie historique. L'auteur examine la réflexion philosophique des scientifiques Emil Du Bois-Reymond, Ernst Mach et Henri Poincaré qui ont tous mis en cause la possibilité, d'une part, d'une épistémologie 
mécaniste où la science pourrait rendre compte d'elle-même par ellemême et, d'autre part, d'un modèle unique de scientificité. Ils ont aussi souligné le rôle des conventions dans la pratique scientifique et la pertinence de l'approche historique pour philosopher sur les sciences. On découvre par ailleurs non sans étonnement qu'un membre du cercle de Vienne, Otto Neurath, a cherché à formaliser l'histoire des sciences pour qu'elle puisse devenir une discipline " that itself deserved the name of science » (17).

La période de l'entre-deux-guerres fut particulièrement féconde dans l'historicisation de l'épistémologie. Des penseurs provenant de traditions différentes programmèrent une épistémologie historique. Rheinberger dresse un parallèle intéressant entre Gaston Bachelard et Ludwik Fleck qui ont souligné la médiation technico-expérimentale et l'enracinement social de toute connaissance scientifique, son caractère collectif et communautaire, la pluralité des savoirs scientifiques de même que l'importance pour l'épistémologue de considérer la pratique effective des sciences et leur histoire, véritable laboratoire de l'épistémologue, comme le dirait Larry Laudan, penseur qui ne figure pas dans le parcours de Historicizing. Bachelard et Fleck s'éloignent d'une conception cumulative de la connaissance scientifique en insistant sur les ruptures qui marquent son développement et qui lui donnent une historicité propre. L'auteur s'arrête sur quatre autres penseurs qui a priori ne semblent pas être liés à l'historicisation de l'épistémologie, mais qui ont néanmoins reconnu l'importance de la question du développement des connaissances. Karl Popper s'est penché sur la question de la dynamique scientifique à travers une analyse de la logique de la recherche. Il récuse le positivisme et délaisse le contexte de découverte, qui relève de la psychologie de la connaissance, pour se concentrer sur le processus formel par lequel on teste la validité de la connaissance. À cette fin, il élabore un modèle déductif de scientificité, à savoir la «falsification " qui prétend que tout énoncé scientifique doit pouvoir être potentiellement réfuté par l'expérience. Edmund Husserl se distancie aussi de l'épistémologie positiviste en plaidant pour une compréhension historique des sciences afin de retrouver l'intention de signification ayant présidé à leur naissance. Activité intellectuelle, le phénomène scientifique ne peut être élucidé que par les sciences humaines, notamment l'histoire. C'est ainsi que, selon Rheinberger, chez Husserl " [e]pistemological grounding and historical explanation must coincide » (42). L'auteur de Historicizing remarque par ailleurs que Martin Heidegger 
a peut-être étonnamment souligné un des leitmotivs de l'épistémologie historique : la science est recherche expérimentale qui, en progressant, se régionalise en différentes sciences ayant chacune leurs conditions procédurales spécifiques. Ernst Cassirer considère, pour sa part, la science comme une forme symbolique. Pour être bien comprise, elle doit faire l'objet d'une histoire culturelle. Seule cette voie permet une compréhension d'ensemble du phénomène scientifique. La possibilité d'une philosophie qui tenterait de systématiser et d'organiser toutes les sciences est mise en cause. Soumettre les sciences au regard historique oblige le philosophe à s'intéresser à la pratique effective des scientifiques, autre mot d'ordre de l'historicisation de l'épistémologie.

L'après-guerre, selon Rheinberger, est marquée par des « attempts at epistemologizing history of science » (51). C'est moins la pratique contemporaine des sciences qui est mobilisée pour une interrogation philosophique que leur développement historique. Quatre penseurs sont examinés. Alexandre Koyré a synthétisé philosophie et histoire des sciences à travers une démarche relevant de l'histoire des idées. Il cerne les présuppositions et les visions du monde orientant le regard scientifique. Thomas Kuhn s'est servi de l'histoire des sciences pour briser leur image officielle, sinon traditionnelle, véhiculée dans les manuels. Le développement scientifique est profondément discontinu, car il est scandé par des révolutions qui transforment la «science normale » ou le «paradigme », concepts kuhniens désignant le modèle régissant la pratique collective des scientifiques. Rheinberger souligne deux éléments ayant souvent échappé aux autres « exégètes » de Kuhn. Kuhn était un internaliste : la pratique scientifique est avant tout déterminée par la communauté des chercheurs et non par une quelconque autorité externe ; et Kuhn ne niait pas le progrès scientifique même s'il soulignait que les différents paradigmes ayant traversé l'histoire des sciences étaient incommensurables. Les sciences progressent à partir de ce qu'elles ont été sans jamais cependant savoir vers quoi elles se dirigent, comme le voudrait une conception téléologique de leur histoire. Stephen Toulmin récuse le logicisme et le formalisme en philosophie des sciences. Ces dernières sont plutôt à ses yeux, selon Rheinberger, " an ensemble of ideas and techniques, whose components and goals constantly change in a mobile intellectual and social environment » (60). Pour être comprises, elles doivent être soumises à une analyse historique fondée sur des études de cas empiriques pour respecter leur évolution diachronique et leur diversité synchronique. Rheinberger 
souligne que pour Toulmin, les sciences ne sont jamais en état de stabilité, leurs fondements sont constamment en question, une thèse que reprendra Pierre Bourdieu pour qui l'enjeu du jeu scientifique est constamment en jeu. L'anarchisme épistémologique de Paul Feyerabend radicalise cette thèse en récusant toute forme de méthodologie normative explicite élaborée par les logiciens. Dans la pratique scientifique effective, ce type de méthodologie est « counterproductive » puisqu'il génère de la confusion et non de la clarté (63).

La prochaine escale du parcours est les années 1960 en France. La figure de Georges Canguilhem y est centrale. Successeur de Bachelard à la Sorbonne, il aura une ascendance sur des penseurs comme Michel Foucault, Louis Althusser et Jacques Derrida, tour à tour examinés par Rheinberger. Canguilhem contribua à l'épistémologisation de l'histoire des sciences. En s'intéressant aux filiations conceptuelles, notamment en biologie et en médecine, il programma une histoire récusant à la fois l'internalisme et l'externalisme en vue d'appréhender la spécificité des sciences dans leur «dialectical unity of continuity and change » (69) médiatisé par leur contexte de production. Rheinberger se penche ensuite sur un autre programme d'épistémologie historique, soit l'archéologie du savoir foucaldienne. Récusant l'histoire des idées et son anthropocentrisme, cette archéologie repose sur trois principes, selon Rheinberger. Son objet est le discours saisi dans sa positivité comme pratique et non les idées auxquelles il renvoie. Elle l'aborde dans son horizontalité synchronique et non dans sa verticalité diachronique. Elle récuse la notion d'auteur pour structurer son appréhension du discours au profit de celle de conditions permettant et limitant son énonciation, conditions formant l'épistémè qui prend la forme d'un a priori historique. Louis Althusser, dans une perspective marxiste, conçoit aussi les sciences comme un mode de production inséparable de son contexte. Rarement cité dans les ouvrages traitant de philosophie des sciences, Jacques Derrida, par ses réflexions sur l'écriture, a participé à l'historicisation de l'épistémologie. Rheinberger montre en effet - et cela constitue une thèse autant originale qu'importante de son essai - que l'écriture est essentielle à la connaissance des procédures pour obtenir de la connaissance scientifique. Derrida a forgé à cet égard le concept d'« historialité » pour penser, comme le remarque Rheinberger, l'« iterative-recursive production of meaning in the irrevocable exteriorization of a generalized writing $»(76)$. 
Dans les « recents developments » (79) de l'historicisation de l'épistémologie, Rheinberger s'interroge sur le programme de l'anthropologie historique des sciences à travers les réflexions de Ian Hacking et de Bruno Latour. Le premier s'intéresse à la pratique scientifique et son « interventionary character» $(80)$. Loin de se réduire à un ensemble de théories représentant la réalité, la science est avant tout expérimentation sur cette dernière. La représentation est également à ses yeux une pratique qui a nécessité la création du concept de réalité pour donner un sens à l'objet des représentations et qui, avec le temps, s'est pluralisée : chaque science se dotant de son système de représentations par lequel elle se constitue. On regrette que Rheinberger ne se soit pas référé à l'ontologie historique (2002) que Hacking programme comme une "méta-épistémologie historique » examinant la trajectoire d'objets " that play a certain role in thinking about knowledge ». Latour aborde le phénomène scientifique comme un anthropologue en prenant pour terrain le laboratoire, son lieu de production par excellence, en vue, selon Rheinberger, d'examiner ce que les scientifiques « actually do in their work, how they proceed when they experiment, how they handle and transform their materials » (83). L'étude des sciences doit se loger sous l'enseigne de ce Latour nomme une « anthropologie symétrique » qui, selon Rheinberger, «puts into question the separation between the natural, the social, and the discursive aspects " (83) des sciences, de même que les catégories (historique, sociologique, philosophique, etc.) divisant leur étude. Hacking et Latour participent d'un «practical turn» (87) de l'épistémologie qui l'amène à se focaliser sur la question des modalités de la résolution des controverses scientifiques et sur celle de l'élargissement du territoire scientifique.

Pour tout dire, historiciser l'épistémologie force à penser le phénomène scientifique moins comme un système abstrait que comme une pratique temporalisée et régionalisée. «La» science est remplacée par des sciences ayant leurs propres normes historiques de fonctionnement. L'épistémologue cherche moins à déterminer ou à prescrire la bonne (et unique) méthode scientifique valable dans tous les contextes qu'elle transcende, qu'à comprendre ce que les scientifiques ont fait, et font effectivement, dans leur pratique quotidienne.

Le mérite de Historicizing réside avant tout dans les confrontations et comparaisons que l'auteur effectue entre les différents auteurs qu'il examine. Celles-ci permettent de mettre en perspective des réflexions 
provenant de différentes traditions ayant concilié philosophie et histoire des sciences. On doit également souligner la concision dont fait preuve Rheinberger pour rendre intelligible un corpus s'étendant sur plus d'un siècle. Enfin, on apprécie qu'il ait étayé son argumentation sur les textes "primaires ", c'est-à-dire écrits par les auteurs en fonction duquel il structure son essai sur l'historicisation de l'épistémologie.

Ce mérite est cependant aussi une faiblesse. Rheinberger ne se réfère pas à l'immense littérature secondaire produite sur les auteurs qu'il a sélectionnés ni même à des recherches s'étant penchée sur l'histoire de l'épistémologie historique ( $c f$. Brenner). On aurait par ailleurs apprécié que l'auteur explicite davantage sa propre démarche pour interroger et thématiser l'histoire de l'historicisation de l'épistémologie, notamment les principes qui ont guidé la sélection des « key texts » (4) balisant son essai. On aurait alors peut-être été en mesure de comprendre en quoi il a pu inclure dans son parcours des auteurs comme Heidegger et Derrida et exclure des auteurs ayant pourtant été des sources majeures de l'historicisation de l'épistémologie comme W. O. Quine avec son programme de la naturalisation de l'épistémologie, Imre Lakatos et sa théorie des programmes de recherche pour rendre compte du développement historique des connaissances scientifiques ou le rationalisme historiciste de la théorie des champs scientifiques de Pierre Bourdieu par laquelle ce dernier cherche à contourner deux écueils de la philosophie des sciences, soit le logicisme essentialise et le relativisme absolu. Ce manque de réflexivité est surprenant, sinon paradoxal, venant d'un praticien de la discipline cherchant à expliciter les démarches scientifiques. L'histoire, comme semblent l'oublier bon nombre d'épistémologues recourant à l'approche historique pour analyser les sciences, est une science au même titre que la physique, la biologie, la chimie ou les mathématiques, qui retiennent en bonne partie l'attention des recherches en épistémologie historique. Bien que l'épistémologie historique, comme le souligne bien Rheinberger, ait consisté en bonne partie en une "epistemologization of the history of science » (3-4), ses praticiens ont mobilisé et continuent de mobiliser la démarche historique sans tenir compte de l'épistémologie des historiens de métier qui, notamment depuis sa transformation disciplinaire au XIX ${ }^{\mathrm{e}}$ siècle, n'ont cessé de s'interroger sur ses préceptes. Ceux-ci ne forment en rien une essence, contrairement à ce que laisse entendre Yves Gingras dans une récente note de recherche du CIRST sur la généalogie et les emplois de l'étiquette "épistémologie 
historique » dans laquelle il nous invite pourtant à « historiciser tout ». L'épistémologie historique, de même que son histoire esquissée par Rheinberger, ne peut se permettre d'ignorer l'épistémologie (historienne) de l'histoire au risque d'une historicisation spontanée des sciences qui pourrait sombrer leur philosophie dans le gouffre positiviste duquel elle voulait la faire sortir, soit celui d'une image ne correspondant pas à la science qui se fait.

Patrick-Michel Noël Université Laval

\section{Références}

Brenner, Anastasios, 2006, "Quelle épistémologie historique? Kuhn, Feyerabend, Hacking et l'école bachelardienne », Revue de métaphysique et de morale 49 (1) : 113-125.

Hacking, Ian, 2002, Historical Ontology. Cambridge, Harvard University Press.

Les cultures du volant $X X^{\ominus}-X X l^{\ominus}$ slècles. Essal sur les mondes de l'automobillisme. Par Mathieu Flonneau (Paris, Edition autrement, 2008, Pp. 220, ISBN 978-2-7467-1 183-9)

Avec cet ouvrage, Mathieu Flonneau ouvre un nouveau champ dans l'histoire de l'automobile en France. En effet, sur le modèle de Wolfgang Schivelbush qui avait, il y a de cela quelques années, proposé une histoire culturelle des voyages en train, Mathieu Flonneau propose d'analyser une histoire culturelle sur la longue durée de ce qu'il appelle l'automobilisme. Selon sa définition, l'automobilisme consiste donc dans «l'ensemble des usages de l'automobile » depuis ses débuts à la fin du XIX siècle jusqu'à l'orée du XXI ${ }^{\mathrm{e}}$ siècle.

L'auteur, agrégé et docteur en histoire, est maître de conférences à l'université Paris I-Panthéon-Sorbonne. Il s'est auparavant intéressé aux mutations sociales introduites à Paris durant le $\mathrm{XX}^{\mathrm{e}}$ siècle dans une thèse pionnière en France et choisit avec cette publication d'élargir son champ d'intérêt. Pour se faire, il s'est appuyé sur une documentation phénoménale et très hétérogène : les ouvrages spécialisés pour chacune des époques (L'Argus), le recours à l'iconographie (Edward Hopper entre autres), les romans (avec de grands noms tels que Marcel Proust, 